\title{
La epistemología del análisis económico estructural
}

\author{
THE EPISTEMOLOGY OF STRUCTURAL ECONOMIC ANALYSIS
}

Mg. Luis Cárdenas (luiscard@ucm.es) Facultad de Ciencias Económicas y Empresariales, Universidad Complutense de Madrid (Madrid, España)

\begin{abstract}
In this paper we focus on the structural approach and structural method developed from Sampedro's work. We propose that this approach is inside the systemic paradigm and it has two interdependent and necessary ideas. On the one hand, we take as a reference some schools of thought in economics and the structural method with two stages (descriptive and synthesis). On the other hand, some macroeconomic principles has been derived from the vision and the methodology, which give the structural approach an analytical, synthetic, aggregate and historiographical character. In this sense, the main argument is that structural analysis has its own macro-theoretical characterization (although eclectic). In this way, the structural approach allows valid analysis in order to increase our knowledge of the economic reality.
\end{abstract}

Keywords: structural analysis, epistemology, macroeconomics, methodology, economic thought.

\section{Resumen}

En este trabajo se realiza una aproximación al enfoque y método estructural, a partir de los conceptos postulados en la obra de Sampedro y su posterior inclusión dentro de un paradigma sistémico, distinguiéndose dos pilares interdependientes y mutuamente necesarios. Por un lado, para tratar los aspectos epistemológicos se distinguen las escuelas consideradas de referencia en economía y se detalla el método estructural basado en la etapa descriptiva y la de síntesis. Por otro lado, se tratan los principios macroeconómicos que se derivan de la visión y de la metodología y que otorgan al enfoque estructural un carácter analítico, sintético, agregado e historiográfico. En este sentido la tesis principal del artículo es que el análisis estructural posee una caracterización macroeconómica propia (aunque ecléctica) que permite realizar análisis válidos de modo sistemático, con el propósito de aumentar los conocimientos sobre la realidad económica.

Palabras clave: análisis estructural, epistemología, macroeconomía, metodología, pensamiento económico.

\section{Introducción}

El pensamiento estructural español (1) tiene su origen en los postulados desarrollados por José Luis Sampedro (1959), quien basándose en distintos autores y escuelas, configura un cuerpo analítico original que ha sido aplicado y ampliado con posterioridad (2). Una idea interesante es la relación entre teoría económica y análisis estructural, desarrollando las definiciones de ambas (Viñas 1976). Esta cuestión fue desarrollada en las primeras décadas de existencia del marco analítico estructural por destacados 
autores (Sampedro, Martínez Cortiña, Beiras, Viñas, Alburquerque), más recientemente, Berzosa (1995) considera que es imposible contraponer los dos planteamientos. Si la teoría económica se define como la ciencia social que estudia la realidad económica, el análisis estructural se encuentra dentro de ella, puesto que es una forma concreta de análisis económico.

Por esta razón es posible proponer como pregunta de investigación cuál es la relación existente entre ambas concepciones, partiendo de que forman parte de un mismo cuerpo analítico. Para responder a esta cuestión se sostiene la tesis de que el pensamiento de análisis estructural contiene un conjunto de elementos de teoría económica que le son propios de forma natural, atendiendo a su origen, y que forman un marco teórico que le sirve como instrumental analítico aplicado. Esta propuesta se fundamenta en unos principios epistemológicos y en unos principios macroeconómicos.

Puede considerarse como teoría económica puesto que está constituida por un conjunto de modelos, entendidos como la combinación, interdependencia y ordenamiento de postulados que permiten desarrollar cadenas de razonamiento lógico con objeto de explicar la realidad económica. Su principal axioma es la adscripción al paradigma sistémico, que puede definirse como el conjunto articulado de ideas que estudian la realidad, económica y social, interpretándola como sistema, i.e., como conjunto coherente y organizado que puede ser explicado reconociendo las interdependencias y jerarquías existentes entre los elementos, la totalidad que configuran y la dinámica que generan (Palazuelos 2000).

Para encontrar los elementos teóricos propios, se analizan las escuelas de pensamiento señaladas por Sampedro (1959) y sus herederas contemporáneas, observándose algunas nociones comunes. En segundo lugar, se considera el método estructural, su relación con otras corrientes epistemológicas y la relación con el concepto aparentemente contradictorio de dinámica. En el apartado siguiente se formaliza en dos etapas de análisis, siguiendo la propuesta de Xosé Manuel Beiras (1987). A partir de las referencias y la metodología se obtienen los principios macroeconómicos inherentes.

\section{El enfoque estructural en un paradigma sistémico}

Un paradigma es un conjunto de proposiciones, normas y reglas de investigación que son compartidas por una comunidad científica en un momento dado. Este marco analítico se configura en torno a una serie de axiomas heurísticos establecidos como principios fundamentales e indemostrables, sobre los que se desarrollan una serie de hipótesis auxiliares contrastables. El paradigma establece la base de cualquier investigación científica, influyendo en sus principales características, i.e., su objeto, hipótesis y método. La vigencia y valoración de los paradigmas dependerán tanto de su capacidad explicativa de la realidad observada, y especialmente de la incorporación de las anomalías (hechos que se encuentran fuera del paradigma), como de los factores sociológicos existentes en el momento de su evaluación.

Se define el paradigma sistémico en economía (Palazuelos 2000) como el conjunto articulado de ideas que estudian la realidad, económica y social, entendiéndola como un sistema, i.e., como conjunto coherente y organizado que puede ser explicado reconociendo las interdependencias y jerarquías existentes entre los elementos y la totalidad que configuran. Esta visión sostiene la concepción del individuo en sociedad, y la existencia de relaciones sociales de producción, e.g., de distribución, de intercambio y de consumo, que conforman la realidad y articulan la sociedad. Siendo, además, sus relaciones de causalidad de tipo circular y no exclusivamente lineal. Esta visión paradigmática (forma de estudiar la realidad económica) puede entenderse como un marco general que comprende distintos enfoques, así lo han señalado Álvarez. et al. (2012), enfatizando sus características propias y distintivas. 
El paradigma sistémico (Palazuelos 2000) considera que el estudio de la sociedad es el estudio de tres componentes: las características socialmente relevantes de los individuos, las propiedades interconectadas del conjunto $y$, especialmente, de la dinámica interna del sistema. Por ello, puede considerarse el resultado de la combinación de interacciones de bucle entre una visión global (una totalidad indisoluble), una visión holística (de individuo en sociedad o ser social) y una visión dinámica (no estaticidad).

Se caracteriza por ser holístico y dinámico. En una definición canónica el holismo sostiene que el conjunto es más que la suma de las partes, principio genético de la macroeconomía, como señaló Keynes: "theory of output as a whole» (Keynes 1936, prefacio), i.e., considera que el conjunto es diferente a los elementos que lo componen al existir relaciones de interdependencia significativas (falacia de la composición). La principal implicación es que no pueden estudiarse los componentes de forma separada al conjunto sin pérdida significativa de información. Pero también se afirma que como los elementos poseen propiedades de permanencia pero no de estaticidad, las relaciones existentes entre ellos provocan un comportamiento dinámico de todo el sistema.

Puede alegarse con razón que el planteamiento sistémico sólo tiene sentido en el análisis de un sistema o de una realidad que puede considerarse como un sistema, pero no lo tiene cuando el análisis se produce en una realidad cuyas relaciones entre los elementos son escasamente significativas, y por lo tanto, el conjunto no tiene características propias. Aunque ciertamente no parece que este sea el caso de la realidad económica, si es posible estudiar algunas relaciones parcialmente, especialmente si lo que se desea son explicaciones limitadas a un ámbito concreto (Martínez González-Tablas 2008).

Siguiendo a Palazuelos (2000) se puede afirmar que el análisis estructural es válido como aproximación al enfoque sistémico por su propia concepción reflejada en el énfasis puesto en la existencia de interdependencias, pero centrándose en las características más determinantes, que se consideran como elementos de articulación del sistema económico. Esta visión de conjunto de la sociedad y las relaciones económicas tiene en consideración el contexto histórico y socioeconómico existente en los hechos observados (Viñas 1976). Asimismo, es un enfoque dinámico, esto significa que explica la evolución de la economía por causas endógenas a la misma, incorporando dimensiones institucionales y sociales.

El término estructura precisa de una especificación pues se suele utilizar en un sentido genérico y en la mayoría de los casos se identifica con organización. En el caso de la economía con organización sectorial o industrial, siendo este sólo uno de los aspectos que se tratan desde el enfoque estructural. Por lo tanto, hay que dotar de significado al concepto de estructura. Dicho término proviene de la palabra latina structūrae, que tiene dos posibles significados, o bien la colocación de un conjunto siguiendo un orden, o bien el resultado de una construcción. Ambos conceptos son válidos como caracterización general del estructuralismo, enfatizando el hecho de que es una consideración metodológica y conceptual, un proceso deductivo y no una realidad en sí misma.

El concepto de estructura y el análisis estructural no es exclusivo de la disciplina económica, siendo originario de la lingüística (Sausurre) y habiéndose extendido posteriormente a otras materias tales como la antropología (Lévi-Strauss), la sociología (Durkheim), la filosofía (Althusser) o las matemáticas (Benacerraf), incluso dentro de la economía no puede señalarse una única materia a la que pertenezca. Todas estas disciplinas comparten la misma concepción de estructura, que se define como las relaciones y aspectos de mayor permanencia. Este tratamiento del término responde a una concepción esencialista, considerando que las estructuras existen por sí mismas, por lo que son universalmente objetivas y no 
mantienen relación alguna con el momento histórico concreto, siendo el objeto del análisis científico descubrir esas estructuras.

Obviamente, esta definición entra en conflicto con la caracterización previa basada en un concepto metodológico y analítico: no existe una realidad objetiva que pueda ser identificada mediante un esquema único, preconcebido, ahistórico y universalmente válido, sino que supone una forma de aproximación a la realidad organizada con objeto de interpretarla como conjunto.

Para realizar la exposición de los principios del estructuralismo, es conveniente distinguir entre varias categorías. Sampedro y Martínez Cortiña (1973) señalan tres concepciones: como método, como enfoque y como disciplina académica. Es posible modificar este esquema discerniendo otras categorías, puesto que en realidad esta distinción es meramente expositiva, ya que son de facto distintas formas de aproximarse a un mismo contenido.

En la categoría ontológica, i.e. de las propiedades del sistema, se pueden distinguir tres niveles analíticos. El primero es el nivel de análisis relativo a la configuración más general de los componentes que constituyen la realidad; en este caso se ha establecido el objeto de estudio dentro del sistema económico capitalista, y por ello, sus rasgos esenciales van a regir en cualquier estructura analizada. El segundo es el nivel propiamente estructural, donde se construye una estructura compuesta de categorías o subestructuras, formadas a su vez por los elementos seleccionados como significativamente representativos y determinantes en los entramados de causalidad. Este escalón es fundamental, puesto que el grado de generalidad anterior debe reducirse para hacer viable el estudio de una realidad económica concreta, por tanto, esta estructura se diseña para entender las pautas y el funcionamiento de la realidad en su conjunto, mediante la combinación de las relaciones y los elementos que configuran el sistema, o parcialmente (Martínez González-Tablas 2008).

En tercer lugar, es necesario considerar el aspecto social (Sampedro 1983) entendido como las relaciones entre los agentes económicos que configuran mediante su interacción y formalizaciones de convencionalismos, el marco sociológico e institucional en el cual opera la realidad económica durante un determinado tiempo y lugar. Asimismo, sobre los vínculos entre las variables que son propias al ámbito económico y las variables de contenido social, aunque es necesario mencionar que no existe una relación unidireccional entre ambas categorías, este aspecto de reciprocidad evita caer en planteamientos deterministas y teleológicos.

La categoría temporal se sostiene en tres planos: (a) el orden acrónico o intemporal representa una entidad estructurada que se puede definir independientemente del lapso temporal en el que se sitúe, i.e., implica unas características que existen fuera del tiempo; (b) el nivel sincrónico o de conexiones simultáneas que refleja una situación de cierta estabilidad, un "estado», como resultado de la estructura subyacente dotada de continuidad; (c) el plano diacrónico o dinámico encarna las propiedades relativas a la estructura de los procesos que transforman el sistema a lo largo del curso histórico (Albuquerque 1981). Por tanto, no existe la contradicción (aparente) entre análisis estructural y análisis temporal; esto se debe a que se distinguen distintos niveles que permiten operar con una mayor profundidad analítica.

En la categoría metodológica, el análisis estructural consiste en delimitar los fenómenos estudiados, establecer los elementos que lo componen y mediante un razonamiento lógico contrastable deducir sus vínculos y la tipología que conforman. Para desarrollar este proceso de estructuración, Beiras distingue entre el modelo descriptivo y el modelo estructural (Beiras 1987:70); el primero consiste en la 
formalización de las primeras etapas del análisis estructural (acotar la realidad sometida a estudio, catalogar sus elementos e investigar y articular) mediante la inducción de los hechos observados. El segundo modelo (denominado por Beiras propiamente estructural) pretende contrastar esas hipotéticas relaciones entre elementos que conforman una estructura. Este nivel requiere de una serie de postulados teóricos que permitan desarrollarlo correctamente. Esta idea será la base del planteamiento del presente artículo.

En la subcategoría formal, es decir, en la concreción explícita del método, el análisis estructural debe incorporar también tres rangos: Primero, es necesario un grado de abstracción teórica mediante la formulación de una serie de hipótesis, supuestos, variables utilizadas y relaciones entre esas variables, con la finalidad de poder contrastar las preguntas de investigación propuestas sobre las características del objeto de estudio. Segundo, es oportuno distinguir las distintas etapas estructuralmente diferenciadas por las que ha transcurrido el desarrollo del sistema económico, para realizar esta diferenciación se recurre al método de periodificación por etapas. Por último, para aplicar ese ejercicio de abstracción a la situación y el contexto histórico concreto es factible utilizar un patrón de crecimiento, mediante el cual se establecen tanto los factores determinantes del crecimiento conjunto, como los factores determinantes y las relaciones causales de las fluctuaciones de aquellas variables consideradas. Asimismo, refleja ese patrón de rasgos característicos (hechos observados) que mejor representan la etapa histórica específica y que permiten obtener una exposición razonada de los hechos (Ramos 2003).

En la categoría del ámbito disciplinar, i.e., la categoría relativa al espacio conceptual configurado por los límites en que se encuadran las cuestiones y los problemas tratados por la disciplina económica, se distinguen dos grandes tradiciones de investigación: la economía (economics) y la economía política (political economy). El análisis estructural debe incluirse dentro de esta última (Viñas 1976), como consecuencia de considerar los vínculos jerárquicos existentes en la sociedad como su objeto de estudio, i.e., al realizarse desde una visión sistémica. Esta categoría permite contextualizar el análisis estructural en las tradiciones de investigación económica.

Por último, una característica fundamental de la disciplina es su carácter firmemente ecléctico (1), que pretende resolver algunas de las cuestiones que han enfrentado a las diversas corrientes económicas. Esta idea se refleja en la metáfora utilizada por Sampedro: "Por eso afirmamos que la Estructura viene a ser como una encrucijada o piedra de clave entre las ciencias económicas. Porque en ella concurren, apuntalándose mutuamente, aportaciones consideradas a veces contrapuestas como el análisis clásico deductivo, por un lado, y el histórico, por otro; lo cualitativo y lo cuantitativo; lo racional y lo no lógico; las dependencias matemáticas funcionales y los lazos institucionales; la Econometría y la Sociología. Y sobre esa bóveda del análisis estructural se alza, en definitiva, un conocimiento científico de la realidad, base a su vez de toda política económica bien concebida, es decir, de la aplicación de nuestra ciencia al progreso y bienestar humano" (1959:242).

En los apartados siguientes se desarrollan algunos principios epistemológicos y de teoría macroeconómica, ambos mutuamente interdependientes, puesto que la teoría económica necesita un método. Se propone que desde la metodología estructural se pueden derivar algunos principios teóricos básicos. 


\section{Principios epistemológicos}

El enfoque de análisis estructural consiste en delimitar los fenómenos estudiados, establecer los elementos que lo componen, y mediante un razonamiento lógico contrastable deducir sus vínculos y la tipología que conforman. La idea central de esta concepción es la búsqueda de los elementos fundamentales, que configuran la trama de conexiones jerárquicas básicas, que mantienen una relación de causalidad circular, con carácter subyacente e irreductible; que los unen y que configuran la realidad económica de forma más estable; permitiendo así representar una realidad estructurada (4). Para desarrollar este proceso analítico es necesario un proceso de estructuración del objeto estudiado, resolviendo la cuestión metafísica de la existencia de una objetividad y la forma epistemológica mediante este proceso (Beiras 1987).

Por un lado integra el contexto histórico mediante la incorporación de las relaciones entre los agentes económicos que configuran, por convención, el marco sociológico e institucional. Por otro lado, se postula un nivel de abstracción teórica que requiere el establecimiento de conexiones entre los elementos considerados, mediante la formulación de una serie de hipótesis, supuestos, variables y relaciones entre las variables utilizadas.

\subsection{Referencias e influencias teóricas}

Las fuentes están tanto en la obra de Sampedro (1959) como en Sampedro y Martínez Cortiña (1973). A efectos expositivos se han reagrupado los autores precursores en una clasificación distinta; además se han añadido escuelas de pensamiento contemporáneo, aunque se mencionan exclusivamente las ideas que sirven para fundamentar los postulados posteriores, y no todas sus contribuciones, puesto que esa tarea excedería ampliamente el objetivo de este apartado.

El primer bloque de referencias históricas lo forman un grupo de distintos enfoques. 1. El enfoque espacial. Una visión espacial implica una ordenación según su situación en el espacio geográfico, algunos autores de esta corriente son Johann Heinrich von Thünen o Alfred Weber. Obviamente esta formalización está profundamente relacionada con la Geografía y en concreto con la Geografía Económica; 2. La concepción anatómica se basa en la enumeración y catalogación de los componentes, en línea con la propuesta de política aritmética o concepción anatómica-aritmética de William Petty. Este autor es pionero de la ciencia económica puesto que inicia el estudio de la economía desde una perspectiva cuantitativa y macroeconómica en su obra de 1672 The Political Anatomy of Ireland; 3. La visión arquitectónica, utilizada por la econometría, especialmente tras la aparición de la contabilidad nacional (Colin Clark, Simon Kuznets y Richard Stone, entre otros), y los modelos matemáticos en macroeconomía.

Desde este enfoque se entiende que la formalización matemática de los modelos económicos analiza la estructura al estudiar las funciones que desempeñan los elementos de la misma. Es el caso de los modelos de Jan Tinbergen y, en especial, las tablas input-output y los coeficientes técnicos de Wassily Leontief; 4. El análisis circulatorio. La visión fisiológica global de los fisiócratas, y en especial, de François Quesnay y la creación del Tableau Economique, predecesor de las tablas input-output, que trataron de dar una explicación al origen del valor en términos naturales, por lo que necesitaban establecer el proceso y las relaciones de producción, i.e., la interdependencia entre los sectores productivos y la demanda agregada. 
El segundo bloque está constituido por distintas tradiciones de autores institucionalistas. En primer lugar, la escuela histórica alemana transcurrió por tres etapas. La que origina la tradición estuvo liderada por Wilhelm Roscher y estableció las bases metodológicas, conceptuales y el programa de investigación. Durante la siguiente etapa, la escuela llegó a ser dominante en la academia alemana bajo la dirección de Gustav von Schmoller, profundizando en el procedimiento científico, no exclusivamente inductivo, de obtener conclusiones válidas. En la última etapa, encabezada por Werner Sombart, se propone el concepto de sistema económico junto con una clasificación de características morfológicas para el estudio de sistemas. En esta época también se pueden incluir a otros autores como su predecesor Friedrich List, o autores formados en este pensamiento como Max Weber. Muchos de estos autores se basan en la sistematización con una visión general; entre ellos se encuentran autores como el propio Sombart que realiza una diferenciación tipológica del capitalismo.

Los planteamientos más interesantes de esta escuela para el análisis estructural pueden resumirse como sigue: (a) la negación que la economía de mercado sea un orden "natural" y, por ello, definitivo, (b) el análisis de las motivaciones humanas mucho más realistas que la ultra simplificada psicología del sujeto movido exclusivamente por el lucro material, (c) el análisis de las fuerzas sociales, acciones colectivas, grupos en acción y demás elementos influyentes en las decisiones que animan la dinámica social, y por tanto, la evolución económica; (d) la integración de los análisis anteriores en estudios morfológicos y tipologías de las formas de vida y de organización social, así como teorías sobre las "etapas" de la evolución económica a lo largo del tiempo.

En segundo lugar, la tradición institucionalista norteamericana, con autores como Thorstein Veblen, John Commons, Wesley Mitchell o John K. Galbraith. En especial, Thorstein Veblen y su "Teoría de la clase ociosa" que cuestiona el racionalismo (o hiper-racionalismo) del homo economicus, integrando al individuo en la sociedad, y por tanto, estudiando su comportamiento individual con las instituciones existentes (inter alia, la emulación pecuniaria y la jerarquía de la clase ociosa), dado que esta interacción entre agentes e instituciones moldea tanto sus pautas de conducta individuales como las propias circunstancias institucionales. Además, la visión dinámica de Mitchell sobre los movimientos cíclicos que caracterizan las fluctuaciones de las economías de mercado.

Tercero, el sociologismo francés, definiendo sociologismo como la inserción del análisis económico cuantitativo en su marco social. Pueden distinguirse dos autores: François Perroux y André Marchal. Perroux fue de los primeros autores que definieron estructura como las proposiciones y relaciones que caracterizan un conjunto económico localizado en el tiempo y en el espacio. Por su parte, Marchal intenta delimitar periodos y límites estructurales mediante el concepto de "toma de conciencia": puesto que el criterio que establece los límites estructurales es totalmente subjetivo, no permite una diferenciación específica reconocido por el propio autor, el cual trata de complementarlo con otro criterio objetivo en base a la consideración del periodo y a la forma de las relaciones, que le lleva a lo que se denomina adaptación estructural.

En cuarto lugar, el institucionalismo sueco, especialmente la figura de Johan Gustaf Akerman (1962), que distingue cuatro acepciones dentro del término de estructura: el concepto histórico, que estudia un conjunto de indicadores de forma cronológica; el concepto económico puro o clásico, dado por el orden natural; el concepto estadístico basado en los datos y las herramientas estadísticas; y el concepto macrodinámico, como formulación de un sistema de ecuaciones simultáneas. La propuesta de Akerman es el concepto estructural de análisis causal (compuesto de 10 ámbitos), basado en la diferenciación de etapas para estudiar los cambios estructurales ocurridos en cada una de las fases, de tal forma que se 
sigue un método dualístico en el que primero se realiza un análisis causal y después un modelo de cálculo basado en el causal. Es decir, primero se especifica en qué clase de sociedad se va actuar y qué variables juegan en ella un papel relevante y solo después se construye el modelo de cálculo, que no pretende tener validez universal, sino que está precisamente elaborado para guiar la actuación en el seno de la sociedad particular de que se trate. En este sentido el análisis causal combina el institucionalismo de Veblen y Mitchell (también Schumpeter) con las técnicas cuantitativas.

Finalmente, entre las referencias clásicas se encuentran las contribuciones de Marx. Este autor puede considerarse como estructuralista al analizar el sistema capitalista y sus relaciones características de mayor duración. La incorporación de los términos de estructura y superestructura (que sólo aparecen una vez en su obra), entendidas como las relaciones de producción que se establecen en una fase del desarrollo en las fuerzas productivas (y la superestructura jurídica, política y formas sociales que se derivan de ellas), es una definición muy restringida del término y dotada de una causalidad unidireccional, puntos que entran en contradicción con las ideas previamente defendidas. Por el contrario, las ideas más interesantes para el tema tratado se pueden resumir en las siguientes: el sistema económico capitalista se define como un sistema de producción de mercancías mediante la propiedad privada de los bienes de producción y la utilización del trabajo asalariado, dirigido a la obtención de beneficios. Se caracteriza por la existencia de relaciones sociales de producción, es decir, relaciones entre los propietarios o los gestores de los medios de producción y los empleados a los que necesariamente han de contratar en el mercado laboral y a los que remuneran salarialmente.

El proceso productivo tiene un carácter mercantil, es decir, la producción de bienes y servicios se dirige explícitamente a su venta en el mercado para la imprescindible obtención de beneficios, lo que provoca un conflicto entre las clases sociales por su participación en la distribución de la renta. El proceso que sigue la producción consiste en tres pasos: la inversión privada por parte de los propietarios del capital determina la capacidad productiva instalada; la gestión de esos medios de fabricación organiza a su vez el proceso de trabajo para transformar el trabajo nominal en efectivo; finalmente, venden el producto del trabajo en el mercado de bienes y servicios. Este recorrido reproduce la acumulación de capital, expandiendo los límites productivos existentes, incrementando el tamaño de las corporaciones, lo que a su vez concentra la propiedad en mercados monopolísticos y centraliza el control del capital. La formación socio-institucional sufre profundas transformaciones al extender el sistema de trabajo asalariado por mantener una reserva de trabajo y transformando continuamente el proceso de producción para aumentar la intensidad del trabajo.

Entre las escuelas contemporáneas de pensamiento económico, como ya señaló Viñas (1976), se encuentran muchos de los herederos de las ideas desarrolladas por las escuelas históricas. Es posible incluir autores neo-marxistas, los radicales norteamericanos y la escuela de las Estructuras Sociales de Acumulación, los institucionalistas tradicionales norteamericanos, los estructuralistas latinoamericanos y los macroeconomistas estructuralistas, los socio-economistas o de sociología económica, la escuela de la regulación francesa, y los autores keynesianos (en la mayoría de sus vertientes) tanto los pioneros de la Escuela de Cambridge, como los autores contemporáneos (5).

No todas las ideas de estas escuelas son compatibles entre sí ni con el enfoque estructural, simplemente son utilizadas como fuentes de utilidad a la hora de obtener un bagaje de conocimiento teórico que permita el desarrollo del método estructural (véase el apartado siguiente). Los puntos en conflicto suelen o bien derivar de alguno de los postulados o bien de las explicaciones concretas a fenómenos observados. Si bien el primer punto tiene interés y es ciertamente fructífero que se contrasten las 
distintas concepciones $y$, en su caso, se lleguen a obtener teorías más sofisticadas mediante la combinación, el enfoque estructural puede aportar respuestas en el segundo aspecto, dado que las explicaciones de los fenómenos observados son su principal objeto de interés.

Un ejemplo representativo es el trabajo de Marglin (Marglin y Schor 1990) que propone un esquema de cuatro componentes (estructura macroeconómica, orden internacional, sistema de producción y reglas de coordinación), esquema que parece encajar de forma adecuada con las nociones fundamentales del análisis estructural. A mi juicio, es bastante acertada la explicación y las motivaciones que expone Gordon para denominar a su modelo macroeconómico como estructural, citando in extenso: "I prefer not to work with some combination of the usual Ricardian/Marxian/Keynesian /Kaleckian/Sraffian labels -whether 'neo' or not- in order to emphasize the synthetic character of the approach represented here, with feet firmly rooted in many of those traditions. I deploy the generic term 'structuralist' for four reasons: (1) to emphasize the structures of determination and effect of economic classes (...); (2) to stress the importance, highlighted particularly by the social structure of accumulation and regulationist approaches (...); (3) to emphasize important communalities with the tradition within the developing world (...); (4) to underscore the inclination of this literature not to insist on explicit micro foundations for every macro relationship it explores" (Gordon 1995:377).

\subsection{El método en el análisis estructural}

En primer lugar, se define el método epistemológico científico como el modo estructurado, organizado y ordenado de obtener resultados, de sistematizar los conocimientos y las conclusiones obtenidas, de acuerdo a unos criterios de reglas lógicas, objetividad respecto a los hechos observados y contraste de la evidencia fáctica. Con esta finalidad se utilizan las hipótesis previamente postuladas basadas en un razonamiento lógico, que mediante su interrelación y combinación, forman una serie de modelos que en su conjunto constituyen una teoría.

La aplicación del método científico requiere de una serie de procedimientos para enunciar claramente la pregunta (o preguntas) que pretende responder la investigación. Para ello se formulan las hipótesis (postulados) fundadas y contrastables con la experiencia para poder responder a esas preguntas, estableciendo las consecuencias lógicas que se derivan de la misma para su posterior contrastación, comprobando su adecuación a los hechos y la confianza que merecen los resultados obtenidos. Es necesario establecer los límites dentro de los cuales es posible afirmar la veracidad de los postulados, así como los nuevos problemas originados por la investigación.

En segundo lugar, se define el análisis estructural como método epistemológico que se basa en su conceptualización de la realidad económica mediante un razonamiento lógico contrastable en un proceso inductivo. En este sentido, se realiza una estructuración de la realidad económica, que consiste en la ordenación de los elementos que la componen en un determinado proceso analítico, pues la configuración otorgada al objeto de estudio no existe en sí misma, sino que es una aproximación heurística de ordenación de los elementos en un conjunto. De tal forma, el conocimiento científico de la realidad económica considera resuelta la cuestión metafísica de existencia de la realidad y la cuestión epistemológica de la facultad de conocer la misma.

La aproximación al realismo científico puede resumirse así: 1) La realidad económica existe y es independiente del sujeto; 2) La realidad en su totalidad es inabarcable por definición como consecuencia de sus características inmanentes; 3 ) Al aplicar el método estructural se obtiene como resultado una 
realidad económica estructurada, que en términos generales será la "estructura económica", que existe en la realidad y puede ser percibida por el investigador; 4) Sobre esta realidad es posible formular hipótesis concretas derivadas de los principios macroeconómicos. En este sentido guarda relación con el "realismo constructivista" defendido por Ronald Giere, al sostener que modelos y sistemas reales, en este caso "estructura económica" y "realidad económica", están vinculadas con un grado suficiente de similitud.

Esto genera una interacción entre el análisis concreto y el esquema pre-conceptual que, en su caso, puede requerir una reformulación para mantener su coherencia fáctica respecto a la estructura económica considerada. Dicho esquema, obtener datos a partir de pre-conceptos para construir la "estructura económica" y su posterior contrastación con las hipótesis, sugiere una interpretación más fuerte del realismo de lo que sostiene Giere. Pero también implica la importancia del bagaje teórico del investigador al proponer las categorías que conforman su esquema, lo que rechaza el sentido más fuerte de realismo y la posibilidad de obtener verdades universalmente válidas (que suscribe un realismo moderado). De tal forma, el criterio de demarcación de su validez científica es su capacidad explicativa, en otras palabras, su objeto es esencialmente explicativo y no predictivo.

Existe una correspondencia entre los principios básicos del análisis estructural y los defendidos por el realismo transcendental, posteriormente ampliado a realismo crítico, desarrollado por Roy Bhaskar, Margaret Archer, Andrew Collier, Alan Norrie o Tony Lawson. Dado que los tres conceptos ontológicos manejados por el realismo crítico, intransitivity (intransitividad), transfactuality (transfactividad) y stratification (estratificación) son aplicados en varios campos, para realizar una comparación con el análisis estructural solo es posible hacerlo en referencia a una realidad ontológica concreta: la realidad económica.

No en vano, Lawson propone denominarlo "realismo estructural", ya que en este punto es donde hay una mayor proximidad al considerar el realismo crítico: "The conception of the social world to be sustained is of a network of continually reproduced inter-dependencies. That is, social reality is conceived as intrinsically dynamic and complexly structured, consisting in human agency, structures and contexts of action, none of which are given or fixed and where each presupposes each other without being reducible to, identifiable with, or explicable completely in terms of, any other" (Lawson 1997:155).

Analizando los principios, en primer lugar sobre la intransitividad, ya se ha comentado que el análisis estructural considera la existencia de una realidad independiente del investigador, pero que para aproximarse a la misma se construyen estructuras mentales a través del método. En segundo lugar, desde este enfoque la economía es por definición un sistema abierto lo que imposibilita el "cierre" o "clausura" del mismo; asimismo no es posible establecer la formulación de leyes universales de orden natural, ya que el objeto de estudio está claramente delimitado. En tercer lugar, la estratificación ontológica es plenamente compartida (véase los tres niveles que propone Sampedro, 1983), de nuevo, como consecuencia de que se entiende la realidad económica enraizada en los aspectos sociales e institucionales y la necesidad de elaborar categorías para el estudio de cada ámbito.

Dos aspectos son los elementos más discordantes con el realismo crítico: las afirmaciones transfácticas de tendencias y el problema de cierre en relación a la econometría. El problema con el concepto de tendencia es que los "enunciados de necesidad natural incondicional" (Lawson 1997:22) se derivan de considerar que la determinación conjunta de los fenómenos pueden ser debidos a causas que no se manifiesten en la actualidad como consecuencia de la apertura del sistema. Con respecto al problema de 
cierre, el punto de partida del análisis estructural es considerar el objeto de estudio y delimitar sus límites, de tal forma que todos los ámbitos considerados deben provocar un cierre heurístico que permite el estudio concreto, ya sea porque considera todos los fenómenos relevantes o porque las causas más lejanas no consideradas actúan mediante esos fenómenos. Esto conlleva también no compartir el rechazo a la econometría de Lawson como consecuencia del problema de cierre. El análisis estructural no rechaza la formalización matemática ni la utilización de modelos econométricos, especialmente los de series temporales (los más frecuentemente utilizados en macroeconomía), sino que los incorporan como un elemento para establecer las relaciones causales subyacentes dentro del procedimiento metodológico, lo que difiere sustancialmente del deductivismo y el instrumentalismo. En definitiva, el realismo crítico y el análisis estructural comparten el elemento de que el objetivo de la disciplina económica es establecer las relaciones causales subyacentes que rigen la realidad económica, su estructura, pero difieren en puntos importantes de su aplicación práctica.

Finalmente, la formalización del método estructural es el denominado análisis estructural, que se compone de distintos pasos en su progreso, como señala Sampedro: "Cuatro son, por tanto, los escalones del análisis estructural: acotar la realidad sometida a estudio, catalogar sus elementos, investigar y articular en un sistema las relaciones que los enlazan y comparar unos sistemas de relaciones con otros" (1959:240).

Esto implica que el primer paso consiste en definir y establecer los límites de la realidad cuya estructura se pretende establecer. Este proceso de delimitación responde al grado de generalidad del estudio y a los límites geográficos y lapso temporal. A continuación, es necesario definir las variables que se van a considerar relevantes para la investigación, este paso es coherente con la concepción anatómica de catalogar los elementos que se consideran como piezas de la estructura.

El siguiente escalón es determinar y establecer las relaciones que articulan funcionalmente a los elementos reales, sujetos y objetos agrupados en las distintas subestructuras propuestas. Este paso puede considerarse como propiamente estructural, ya que se establecen las relaciones fundamentales de los sujetos colectivos (como resultado de su agrupación) y las distintas magnitudes macroeconómicas utilizadas en el proceso. Una vez obtenida la estructura que mejor representa a la realidad económica, se analiza mediante un patrón de rasgos característicos de la realidad.

El paso de caracterización será diferente en función del grado de focalización que se utilice y cabe distinguir tres niveles analíticos. Primero, si lo que se realiza es el estudio de una subestructura concreta, se establecen sus principales particularidades en una representación formalizada como resultado de un análisis parcial. Por el contrario, si se abarca una estructura en su conjunto, el paso consiste en establecer un modelo o régimen de crecimiento, que se define como fenómenos formalizados por la elaboración de modelos macroeconómicos que reflejan las pautas de comportamiento características de una determinada estructura económica. Estos modelos permiten personificar la etapa histórica sobre la que transcurre la realidad observada, por esta razón el régimen es relativamente estable pero no inmutable. Por último, se realiza la construcción de una tipología que permita comparar las estructuras diferentes, sea en el plano temporal o en el plano geográfico; este paso permite que las tipologías y los resultados que se derivan de ellas sean susceptibles de contrastación.

Para desarrollar este proceso de estructuración, Beiras distingue entre el modelo descriptivo y el modelo estructural; el primero consiste en la formalización de las primeras etapas del análisis estructural (acotar la realidad sometida a estudio, catalogar sus elementos, investigar y articular) mediante la inducción de 
los hechos observados. El segundo modelo, denominado "propiamente estructural" (Beiras 1987:70), pretende contrastar esas hipotéticas relaciones entre elementos que conforman una estructura. Este nivel requiere de una serie de postulados teóricos que permitan desarrollarlo correctamente.

A partir de la obra de Sampedro (2010), es adecuado añadir (en sentido estricto modificar) una dovela a la bóveda (siguiendo con la metáfora) que configura el estructuralismo mediante la encrucijada de los distintos enfoques. Siguiendo esta idea y la propuesta por Beiras (1987), se proponen dos etapas a la hora de desarrollar el método de análisis estructural, en referencia al plano analítico en el que se apliquen la etapa descriptiva y la etapa de síntesis, basados en esquemas de relaciones económicas. Este aspecto se desarrollará en el apartado 4, dedicado a la aplicación del método mediante dos etapas.

\subsection{La dinámica macroeconómica y el análisis estructural}

En la categoría temporal, el análisis estructural analiza las fluctuaciones siguiendo la definición de "dinámica macroeconómica". La palabra dinámica, en su origen etimológico griego, dynamos, se puede traducir como fuerza o potencia. En la actualidad se define como "perteneciente o relativo a la fuerza cuando produce movimiento" o como "sistema de fuerzas dirigidas a un fin". Así, la dinámica macroeconómica surge como consecuencia del comportamiento del sistema de fuerzas estructurales en movimiento, dirigidas a su vez al proceso de acumulación y que se formaliza en ciclos económicos bajo una perspectiva macroeconómica. De esta forma, es posible combinar en una única definición de dinámica los conceptos aparentemente antagónicos de estructura (como lo más permanente) y ciclo (como lo más volátil). En otras palabras, el plano sincrónico de correspondencias simultáneas con el diacrónico de interacción en movimiento.

La idea fundamental es el análisis diacrónico basado en la estratificación histórica, es decir, el estudio del proceso económico a través del tiempo mediante el reconocimiento de las épocas históricas específicas que condicionan la evolución del sistema económico en su transformación evolutiva. Asimismo, en estas etapas se produce una concatenación de fases de crecimiento y recesión, i.e. ciclos, que determinan de forma endógena, pero sujeto a shocks aleatorios, el proceso de desarrollo de largo plazo. Esta visión es compartida por muchos de los autores que se han considerado como referencias teóricas.

Por ello, el enfoque macroeconómico estructural, realizado en retrospectiva, consistente en un proceso de estructuración por "etapas". Se basa en establecer una serie de articulaciones entre elementos que configuran una estructura relativamente homogénea, es decir, considerar que es posible representar la realidad económica mediante una determinada estructura, en un período temporal y una zona geográfica concreta. Este plazo temporal es considerado como una etapa en el proceso de desarrollo económico. Como consecuencia, los hechos observados no son necesariamente idénticos en todas las etapas; de hecho es lógico que varíen en función de su contexto histórico.

La utilización del concepto de hechos observados o "estilizados" conlleva la búsqueda de los aspectos más importantes para comprender una realidad histórica, implícitamente se deduce que son ateóricos, o al menos preteóricos, es decir, directamente observables en la realidad mediante las técnicas empíricas. De esta forma se establecen los rasgos característicos de la realidad económica que debe ser explicada por la teoría, dicho de otra forma, la teoría debe procurar explicaciones sobre el origen y evolución de las principales características de la realidad económica. La obtención de los hechos, i.e., los rasgos característicos de la realidad económica que constituyen una serie de patrones o regularidades, requiere algún tipo de método de aproximación para obtener resultados coherentes que formen un objeto de 
análisis y no un conjunto de datos. En términos metodológicos, anteriormente se ha denominado modelo descriptivo estructural a este nivel analítico.

Asimismo, en las distintas etapas del crecimiento pueden articularse los hechos observados mediante una serie de patrones, es decir, una serie de regularidades que caracterizan la trayectoria de su evolución. Estos patrones son susceptibles de comparación entre países y épocas, para obtener las similitudes y diferencias que configuran el curso de su evolución. A este conjunto interrelacionado de pautas se le denomina modelo o régimen de crecimiento, que se define como fenómenos formalizados por la elaboración de modelos macroeconómicos que reflejan las pautas de comportamiento económico características de la realidad estructuralmente analizada. Cada estructura puede ir asociada a un régimen de crecimiento similar o diferente del existente anteriormente, aunque si el cambio ha sido sustancial, en general, determinará la creación de un nuevo régimen. Por tanto, su análisis debe ir acompañado de un modelo capaz de interpretar esas observaciones para poder caracterizar cada etapa, en otras palabras, un modelo sinóptico estructural.

La periodización puede entenderse también como una clave de bóveda entre el análisis de distinción temporal, es decir, un nexo entre el nivel de estudio relativo al largo plazo, i.e., en el que se producen grandes transformaciones; y el nivel de corto plazo, cuando las dotaciones de factores están dadas. Pero además combina el plano de la abstracción teórica (deductivismo) con la interpretación meramente empirista (inductivismo), es por tanto, la intersección entre el plano temporal (corto y largo plazo) y el análisis aplicado.

Finalmente, en el plano temporal sincrónico (simultáneo) se sustituye el principio de equilibrio por el de "estado", es decir, una situación de cierta estabilidad resultado de la estructura subyacente. Desde los tres tipos de equilibrios establecidos (instantáneo, intertemporal y estacionario) es posible desarrollar este principio. Primero, las relaciones de igualdad observadas entre las variables en el corto plazo no implican que el estado sea de equilibrio instantáneo, sino una consecuencia de la formulación realizada. En general ese resultado es siempre ex-post o son identidades útiles para interpretar una situación o período concreto, como en el caso de la contabilidad nacional.

Respecto al equilibrio intertemporal es difícilmente asumible desde una perspectiva que no incluya agentes optimizadores con expectativas racionales. Por el contrario, desde este enfoque se sostiene que el tránsito de un estado a otro se produce sobre la tendencia histórica, caracterizada por los elementos más estáticos o de mayor permanencia durante una etapa histórica estructurada. Por último, el equilibrio estacionario es asumido como un instrumento analítico, necesariamente endógeno desde estos postulados. De existir una tasa de crecimiento natural, esta se deriva del conjunto de elementos que configuran la estructura del proceso evolutivo. En resumen, el concepto de intertemporalidad ha sido sustituido por el de dinámica.

Las concepciones aquí propuestas guardan semejanzas con los planteamientos de Martínez Cortiña, así como de Alburquerque (1981), denominadas como análisis dialéctico-estructural. Esto implica que desde una visión global de la realidad, el aspecto dialéctico destaca la permanente transformación y evolución de la realidad económica. Por un lado internamente entre los propios componentes estructurales y, por otro lado, externamente entre diferentes estructuras consideradas. Por tanto, incluye el aspecto teórico consistente en la construcción conceptual de modelos explicativos de la realidad observada empíricamente, que permitan profundizar en el conocimiento de la misma. Por último, se puede concluir que es necesario entender el análisis estructural en su sentido dinámico, es decir, la evolución de los 
procesos económicos (y del conjunto de variables involucradas) tiene que ponerse en relación a la propia evolución del marco institucional y a las interdependencias, su sensitividad y transformación a lo largo del tiempo. En palabras de Sampedro y Martínez Cortiña: "Sólo mediante la integración de ambas visiones de lo real -el rigor cuantitativo y el sentido histórico - podrá avanzarse hacia el horizonte del futuro, apoyándose a la vez en los dos correspondiente métodos básicos: el análisis formal de las estructuras y la visión dialéctica de los procesos" (1973:288).

\section{La aplicación del método mediante dos etapas}

Entendiendo la teoría como un conjunto de hipótesis cuyas consecuencias se aplican en el análisis económico, y que sirven para relacionar determinado orden de fenómenos, la modelización consiste en un proceso deductivo, una abstracción de la realidad (el denominado "vicio ricardiano"), que representa el comportamiento de un sistema lógico mediante la propuesta de una serie de hipótesis, que combinan las variables dependientes y explicativas estableciendo las relaciones existentes entre ellas. Su formalización debe ser coherente con la necesidad de que sus hipótesis y resultados sean contrastados con la realidad. En el análisis estructural el proceso de modelización no obedece a un ejercicio meramente deductivo, sino que consiste en un proceso de estructuración de la realidad económica, pero también en un proceso de interpretación y de explicación de esa realidad. El objetivo último es construir un relato razonado de los hechos considerados. En palabras de Ramos (2003), desarrollar explicaciones históricas de naturaleza cronológica, concreta y multicausal.

\subsection{Etapa descriptiva}

La mera observación empírica no es suficiente para comprender la realidad. Puesto que cada descripción implica interpretación, es necesaria otra herramienta de análisis científico. Por lo tanto, se aplica un esquema teórico del sistema económico elaborado con el objeto de facilitar la comprensión y el estudio del mismo, reflejando las primeras etapas del análisis estructural: acotar la realidad sometida a estudio, catalogar sus elementos e investigar y articular las relaciones existentes en los hechos observados.

La formalización del nivel descriptivo consiste en distinguir las categorías conceptuales, que deben ser entendidas como subestructuras, en las que se incluyen una serie de variables representativas y determinantes en los entramados de causalidad. Cada subestructura puede ser definida como una unidad completa pero interdependiente, puesto que la red de relaciones entre los elementos son de mayor significatividad que las mantenidas con otros elementos. Por tanto, los nexos entre componentes de distintas subestructuras son de menor significatividad y los vínculos no determinan al mismo nivel de importancia, aunque sin duda existen.

Por ello, las interdependencias pueden ser de dos tipos: interdependencia directa o fuerte (cuando los elementos son indisolubles para el análisis estructural) e interdependencia indirecta o débil (cuando esos vínculos pueden obviarse en el análisis parcial o en el análisis estático), pero en ningún caso en el análisis general de la realidad estructurada. Esta distinción es claramente analítica y consecuencia de un desarrollo lógico, pues las relaciones entre categorías económicas de la misma naturaleza son más relevantes. A esta clasificación de interdependencias es necesario añadir las analizadas en la etapa de síntesis, las relaciones estructurales, entendidas como los vínculos más estables y significativos entre una serie de elementos o variables (conceptos económicos identificados mediante variables). 
A diferencia del primer tipo de interdependencias, estas relaciones podrían considerarse existentes en términos ontológicos, al ser el resultado de una observación empírica previamente aceptada y no como consecuencia del método utilizado. En realidad, establecer los patrones reiterativos es el resultado de una comprensión analítica. Por ello, esta forma de interdependencias debe ser incluida en la etapa de síntesis como método de análisis parcial, basando su existencia en regularidades empíricas previas explicadas en términos de deducción lógica a partir del análisis explícito.

Desde este nivel descriptivo se limita la realidad estudiada, en términos geográficos y temporales, asimismo como las variables utilizadas. Posteriormente se establecen las relaciones existentes entre los elementos, sean esas relaciones internas en las secciones o entre distintas categorías. Este esquema de subestructuras se puede componer de tantos elementos como se crean necesarios, pero hay que considerar que un análisis demasiado agregado perderá capacidad explicativa y un análisis excesivamente desagregado será inoperante. Por esta razón, es adecuado distinguir seis subestructuras (aunque este esquema no es exclusivo): la oferta productiva, la demanda agregada, la distribución de la renta, la estructura monetaria y financiera, la estructura social y la inserción exterior en la estructura internacional.

La primera de estas subestructuras es el aparato productivo, la capacidad de producción instalada. Las variables que incluye son la dotación de recursos, i.e., los recursos naturales, el medio físico y geográfico, el nivel de desarrollo técnico y tecnológico. El stock de capital productivo y el stock en infraestructuras, el tamaño de la población, la oferta de trabajo, la productividad, la organización sectorial y el grado de utilización de la capacidad productiva. Aunque en un momento concreto las dotaciones están dadas, con el tiempo se modifican sustancialmente de forma endógena: la capacidad productiva, el nivel de progreso técnico y el nivel tecnológico.

La demanda agregada se constituye mediante las variables establecidas en la contabilidad nacional. Estas son el consumo privado en bienes y servicios, la inversión pública y privada, el nivel de gasto público, el volumen de las importaciones y las exportaciones, consideradas conjuntamente como exportaciones netas, y como variable de ajuste las existencias disponibles. Las variables que la forman mantienen una serie de conexiones, así puede establecerse que la proporción dedicada al consumo (público y privado) depende del nivel de renta alcanzado. El vector integrador es la demanda de inversión, que juega un papel central en la evolución de la economía al combinar la oferta y demanda de fondos prestables. De esta forma vincula el ámbito de la macroeconomía con la microeconomía mediante las variables condicionantes de la toma de decisión, donde la rentabilidad es de la mayor importancia. Asimismo, une al sector productivo de bienes y servicios con el sector financiero. Además, es el puente entre el corto y el largo plazo, al ser el determinante del proceso de acumulación de capital.

La distribución de la renta tiene importantes consecuencias en la evolución económica. Desde una desigualdad por origen funcional de la renta pueden distinguirse entre las rentas del capital y las rentas del trabajo. La proporción en su reparto va a condicionar la demanda agregada y los efectos del ciclo económico. La distribución de la renta depende entre otros factores del grado de monopolio alcanzado, de la situación del mercado laboral o de la capacidad redistributiva del Estado.

La estructura monetaria y financiera se divide en (a) el ámbito monetario, con sus variables representativas de masa monetaria, los tipos de interés en el mercado monetario y oficial, las cotizaciones de los mercados primarios de valores y el tipo de cambio, en definitiva las relativas a la política monetaria, y en (b) el ámbito financiero, que depende de la morfología del sector, el volumen 
crediticio, así como su origen y destino, el volumen de activos financieros y los tipos de mercado interbancario. La distinción entre ámbitos es meramente formal. Existe una relación de retroalimentación entre el ámbito monetario y el financiero mediante los mecanismos de transmisión de la oferta monetaria y una relación con el sector productivo al existir endogeneidad de la oferta monetaria mediante los canales crediticios.

La inserción exterior en la estructura internacional corresponde al grado de apertura, la tipología de los productos comercializados, el papel de las empresas transnacionales, el destino y origen de sus operaciones comerciales y financieras, y el saldo resultante de la balanza de bienes físicos y financieros. Desde mediados del siglo XX, y especialmente desde los años 80 , las economías nacionales no pueden ser estudiadas sin considerar el proceso de mundialización que ha transformado e influido en el resto del ámbito, especialmente en los procesos productivos, la composición sectorial, el tamaño y capacidad de las empresas multinacionales (la morfología de los mercados) o las finanzas internacionales de la economía mundial.

Por último, la estructura social se define como el marco institucional que constituye el conjunto de relaciones convencionales, jerárquicas e históricas y que configura un determinado marco de articulación y estratificación social, i.e., los elementos sociales, culturales, jurídicos y políticos en el cual se producen las decisiones y comportamientos de los agentes económicos. Es inmediato comprobar que es en este punto donde se observa en mayor medida la influencia del institucionalismo y sus contenidos teóricos. Las relaciones entre los elementos son: las relaciones entre los trabajadores y empresas (organización productiva), las relaciones entre los propietarios y gestores del capital, i.e., las formas de competencia y gestión corporativa, el papel de los poderes públicos e institucionales, en concreto las políticas públicas y la formación social dominante. Por último, la relación entre los trabajadores, en concreto la organización laboral y su capacidad de negociación salarial. Las relaciones del mercado de trabajo están sujetas a la regulación laboral, las formas de contratación, remuneración, entradas y salidas del mismo y la organización dentro de la empresa. La existencia de correspondencias teóricas entre la estructura social y la realidad económica son de carácter circular, no establecen una unidireccional estricta.

\subsection{Etapa de síntesis}

Por otra parte, un modelo de sinopsis estructural o etapa de síntesis-estructural, se define como un sistema lógico basado en relaciones hipotéticas que combinan variables dependientes y explicativas cuya formalización de los postulados debe ser coherente con los resultados del modelo descriptivo. Consiste en la exposición general de las relaciones de interdependencia y jerarquía expuesto en sus líneas esenciales. Haciendo posible contrastar los hipotéticos vínculos entre los elementos y variables seleccionadas, con objeto tanto de explicar el comportamiento del conjunto de la realidad estructurada mediante un determinado régimen de crecimiento, como de procurar interpretaciones de relaciones específicas.

Como señala Sampedro (2010), al construir una representación simplificada de la realidad económica se establece la necesidad de desarrollar un modelo, y concretamente, la necesidad de desarrollar un modelo econométrico para su contrastación empírica. Este no es más que otra posibilidad de progresar en una investigación y dependiendo de su objeto puede ser de mayor o menor utilidad, teniendo un carácter más confirmatorio que de descubrimiento. En este marco, es necesaria cierta formalización matemática del modelo para establecer mejor las complejas interacciones e independencias que en un 
análisis exclusivamente discursivo. Por lo tanto, primero se establecen las conexiones entre los elementos y a continuación se proponen unas hipótesis susceptibles de formalización matemática y de estimación econométrica utilizando las series económicas disponibles.

Es necesario aclarar que la propuesta sintética realizada incluye una serie de modificaciones ma non troppo, las dos etapas heurísticas propuestas, y tienen una fuerte vinculación con los planteamientos de Sampedro (1959). Al seguir la idea esencial de Sampedro (1983) es fácil trazar los lazos que unen ambas propuestas. El primer modelo puede ser considerado la superación del enfoque exclusivamente "anatómico" por uno sistematizado mediante subestructuras formadas por los elementos representativos, y el segundo modelo continua en la perspectiva del enfoque "arquitectónico", en el sentido de una formalización matemática de los modelos macroeconómicos que analiza la estructura construida al estudiar las relaciones y funciones que desempeñan los elementos dentro de la misma.

En la misma línea, el método para desarrollar la estratificación de las etapas históricas se basa en dos pasos. Primero se examinan una serie de indicadores estadísticos con el objeto de distinguir las distintas fases y para ello es necesario establecer un criterio de división de las etapas a priori. Posteriormente, se analizan las principales macro-magnitudes que constituyen la parte esencial del proceso económico estudiado y se vinculan al marco socio-institucional, denominado como estructura social. La transición entre las etapas se produce por cambios estructurales, entendidos como modificación sustancial de la articulación básica entre los elementos fundamentales de la estructura económica. Estas transformaciones requieren un análisis multicausal, a su vez da lugar a una severa interrupción de la senda de expansión de la acumulación y crecimiento de la economía.

Una vez aceptado este esquema metodológico, se hace imprescindible incorporar elementos teóricos que configuren el paso sinóptico, puesto que sin ellos el análisis estructural planteado en estos términos estaría incompleto. En el apartado siguiente se desarrollan los conceptos económicos teóricos que se derivan del enfoque estructural y que son necesarios para desarrollar el método.

\section{Principios macroeconómicos}

A priori puede parecer que este enfoque no provee de los fundamentos teóricos para establecer las relaciones entre esas variables y los razonamientos explicativos que se derivan de ellos. Pero a partir de los elementos característicos del núcleo conceptual (conjunto axiomático inherente), es posible dilucidar algunos principios económicos inmanentes en su concepción epistemológica. Observando las fuentes de pensamiento de las que bebe el análisis estructural, se pueden deducir las características esenciales que estas corrientes aportan al análisis estructural. Por un lado, el énfasis en la importancia del método utilizado de fuerte carácter aplicado y, por otra parte, el elemento común de interpretar la realidad mediante el establecimiento de los aspectos más importantes y la posterior construcción tipológica en sus términos esenciales.

En primer lugar, el axioma fundamental y diferenciador del análisis estructural es considerar que la realidad ontológica puede explicarse mediante un proceso de "estructuración", i.e., la formulación de un esquema teórico de ordenación de los elementos de un conjunto. Esta idea puede aplicarse a distintas realidades (el proceso de estructuración no se suscribe necesariamente a un único objeto de análisis), pero su formalización más habitual es considerar el conjunto, la "estructura económica". 
Una vez establecido el primer punto, es inmediato observar el carácter necesariamente ecléctico de los principios teóricos que acompañan al proceso epistemológico, dado que es imposible establecer a priori en todo momento y lugar las relaciones de causalidad entre los elementos considerados. Esta idea no significa que no existan una serie de regularidades empíricas y observables que permiten la construcción de esquemas o patrones de comportamiento, como los que se han defendido en el apartado anterior.

El principal concepto teórico es considerar la demanda agregada como el condicionante estructural, lo que de facto supone aceptar el "principio de demanda efectiva". Este se define como la demanda agregada respaldada por capacidad de pago y de compra que dirige la actividad económica, i.e., establece que la producción se ajusta a la demanda. Si bien es un principio apriorístico no es en sentido estricto un axioma, puesto que se deduce de la orientación al mercado de la producción y no al autoconsumo. Por lo tanto, solo es válido a la hora de analizar sistemas con predominio del mercado en la formas de distribución y de destino de la producción. Del principio de demanda efectiva se desprende que no existe ninguna razón por la cual la economía ha de tender al equilibro, a la plena utilización de factores, y con ello al pleno empleo. Este principio rige sobre distintos aspectos de la macroeconomía como son, inter alia, la inversión, el empleo o el dinero y provee de un bagaje teórico a la hora de realizar estudios concretos.

Otro condicionante es la visión sistémica. El "paradigma sistémico" establece la visión general y la concepción de la realidad; considera que el estudio de la sociedad es el estudio de tres componentes: las características socialmente relevantes de los individuos, las características propias e interconectadas del conjunto y la dinámica del sistema. Por ello, puede considerarse el resultado de combinar una visión global (una totalidad indisoluble), una visión holística (de individuo en sociedad o ser social) y una visión dinámica (no estaticidad).

Como el sistema opera con un determinado conjunto de instituciones, es necesario diferenciar el momento histórico sujeto al análisis y su evolución, condicionada por la característica evidente de que el tiempo solo tiene una dirección. Debido a esto en ocasiones se denomina enfoque histórico-estructural. La historia es entendida como una trayectoria única e irrepetible, determinada por fuerzas endógenamente generadas dentro de la propia dinámica del sistema sobre el que se producen shocks estocásticos e impredecibles. Por lo tanto, se deduce que la dinámica tiene un carácter inherentemente cíclico y heterogéneo, permitiendo entender el presente como historia. Es dinámica porque analiza la estructura de los procesos, puesto que tanto los elementos como sus relaciones varían a lo largo del tiempo. Estos procesos no son exclusivamente aleatorios, sino que tienen un curso histórico, en el sentido de especificidad histórica y no meramente cronológica.

De igual forma, desde los principios epistemológicos y los cuatro principios previos, se derivan otras características. Respecto a la formalización, establece la necesidad de verosimilitud en los postulados propuestos en relación con los hechos observados, i.e., la teoría no puede construirse en el vacío. Esta propiedad no implica que se realice una teorización ad hoc para explicar los hechos, sino que tiene que mantener un vínculo con la realidad que pretende interpretar.

Asimismo, no se trata de una modelización basada en el instrumentalismo, entendido como la concepción que considera la validez de los modelos por sus resultados y no por su verosimilitud. Por el contrario, en el análisis estructural se deriva que los modelos son útiles en la medida que sirven para la interpretación y explicación de la realidad. Esto implica un carácter de tipo sintético. Como todo modelo es una representación simplificada, desde esta perspectiva ha de buscar las líneas esenciales e 
irreductibles del objeto de estudio, puesto que el enfoque estructural tiene por objeto la búsqueda del entramado de relaciones causales subyacentes y circulares entre los elementos de un conjunto y, a partir de ahí, la construcción de narraciones argumentadas sobre los hechos considerados en un determinado momento histórico (Ramos 2003).

El enfoque estructural establece una perspectiva agregada, i.e., se parte del análisis general al parcial o concreto. La aplicación práctica es mediante la formulación de modelos que se construyen e inciden en las relaciones agregadas. Por tanto, no establece explícitamente los fundamentos individuales que corresponden al comportamiento general, puesto que solo se consideran los aspectos socialmente relevantes y no la representación general en la toma de decisiones individual. De esta forma, no se incurre en una falacia de la composición, i.e., las propiedades de un elemento difieren de las propiedades del conjunto, o en otros términos, los resultados esperados por el comportamiento de un elemento no son representativos de los resultados del conjunto.

Este concepto supone una "macrofundamentación" de lo particular. Desde el principio de estructura no es admisible la unidireccionalidad del nivel micro al macro, por ello, el sistema competitivo y la formación de precios no es independiente del escenario macroeconómico, de hecho, va a estar determinado en gran medida por su situación a nivel agregado. Entre los factores productivos destacan cuestiones como la organización sectorial, la inserción exterior de la economía, la acumulación de capital, el tamaño empresarial y las formas de organización o la infrautilización de la capacidad productiva. Como resultado, los mercados de bienes se encuentran inherentemente en competencia monopolística, lo que se traduce en la existencia de poder de mercado y los precios son establecidos por distintos métodos de aplicación de márgenes.

Sobre el comportamiento de los agentes económicos, la concepción holística implica entender al individuo como un ser social, por tanto no se puede interpretar de forma independiente al sistema del que forma parte, i.e., sin considerar cuestiones como el modo de producción, la clase social, las relaciones y jerarquías existentes o la cultura. Para reflejar este comportamiento se sostiene que en un entorno de incertidumbre los individuos toman decisiones basadas en reglas de comportamiento heurísticas, formadas en función del entorno social o las preferencias desarrolladas mediante las experiencias cognitivas, en una palabra, instituciones. Por tanto, se sostiene una visión "institucionalista" del comportamiento individual. Considerando que gran parte de las referencias consideradas previamente forman parte del institucionalismo en sus distintas vertientes, no es difícil incorporar su concepción de "instituciones".

Desde la escuela institucionalista, se definen como un conjunto de hábitos y costumbres convertidos en reglas o pautas de comportamiento al ser aceptadas por un colectivo. El hábito se define como la inclinación a seguir un patrón de comportamiento previamente adoptado que no requiere de reflexión previa, factible en sí mismo y que se basa en la repetición. El hábito configura el marco en el que se desarrolla el proceso cognitivo y la incertidumbre inherente sobre las situaciones futuras se atenúa mediante estos patrones. Todas las instituciones se caracterizan por ser la interacción entre agentes y de los agentes con las instituciones. Se sostienen por existir concepciones comunes, por ser duraderas al reforzarse y legitimarse en sí mismas. Las instituciones no son homogéneas (es habitual distinguir entre formales e informales), establecen un carácter funcional, idiosincrático y un orden jerárquico mediante la asignación de un estatus al elemento institucional. La principal utilidad de las instituciones es reducir la incertidumbre esencial sobre el tiempo futuro, permitiendo establecer rutinas de comportamiento en prospectiva y fácilmente predecibles, que pueden mejorar mediante la experiencia y el aprendizaje. 
En resumen, los modelos macroeconómicos coherentes con la epistemología estructural deben estructurar la realidad siguiendo un esquema teórico con las siguientes características:

Carácter analítico. Verosimilitud de los postulados, contrastación permanente con los hechos observados (no teoría ad hoc), concepción interpretativa (no instrumentalista), énfasis en la explicación y en narraciones argumentadas.

Carácter sintético. Es sistemático y no existe una división plena entre inducción y deducción, representación de la realidad en sus líneas esenciales, búsqueda del entramado de relaciones causales subyacentes y circulares, construcción de tipologías y su posterior comparación.

Carácter agregado. Evita falacia de la composición, no establece explícitamente los fundamentos del comportamiento individual, macrofundamentación de lo particular, incorporación del principio de demanda efectiva y sus corolarios.

Carácter historiográfico. Idea del presente como historia (causación acumulativa), negación de la existencia del orden natural, transformaciones de los elementos sociales y jerarquías, dinámica endógena de los procesos y las etapas.

\section{Conclusiones}

En este trabajo se ha defendido el enfoque estructural en economía, enmarcado en un paradigma sistémico y basado en dos pilares necesarios y complementarios (principios epistemológicos y principios macroeconómicos), dentro de la tradición keynesiana, institucionalista y socio-económica. Se sostiene que la teoría económica requiere la existencia de un método para su desarrollo, por tanto desde el lado epistemológico es posible desarrollar dos etapas en el proceso epistemológico en función de su nivel analítico. Una etapa descriptiva que permite la caracterización y delimitación de la realidad económica y un nivel sinóptico de explicación de esas observaciones en sus líneas esenciales.

Adicionalmente, en el plano macroeconómico el enfoque estructural incluye necesariamente los preceptos de carácter analítico, carácter sintético, carácter agregado y carácter historiográfico. Como además implica la aceptación de facto del principio de demanda efectiva, al ser la principal condicionante estructura y la incorporación de jerarquías sociales, tiene un fuerte bagaje teórico que bebe de distintas fuentes, o en otras palabras, es esencialmente ecléctico.

A pesar de que estos elementos subyacían en la mayoría de los análisis estructurales, su especificación y enumeración suponen una aportación significativa. Se ha señalado su relación con algunas escuelas de pensamiento y una parte de la literatura económica contemporánea, especialmente del estructuralismo latinoamericano. Una futura línea de investigación puede desarrollar y comparar las características comunes y divergentes.

En resumen, el enfoque estructural en un paradigma sistémico presenta un cuerpo teórico propio y posee las suficientes herramientas analíticas para desarrollar distintas subteorías interpretativas en función del tema tratado. 


\section{Notas}

(1) En este artículo se aborda exclusivamente la tradición del estructuralismo económico desarrollada en España, aunque a mi juicio algunas ideas son compartidas con el estructuralismo latinoamericano. En esta tradición se encuentran, inter alia, los siguientes autores: José Luis Sampedro, Rafael Martínez Cortiña, Ramón Tamames, Ángel Viñas, Xosé Manuel Beiras, Francisco Alburquerque, Enrique Palazuelos, Martínez González-Tablas, Carlos Berzosa, José María Vidal Villa y Javier Martínez Peinado.

(2) Ejemplos recientes de la aplicación del análisis estructural al estudio de la economía mundial es Palazuelos (2015).

(3) Como señala Palazuelos (2000:123): “No es una disciplina que se afirme en contraposición de la Teoría Económica, sino que reclama la necesidad de situar sus referencias teóricas, del mismo modo que lo hace con respecto a la historia económica, la política económica, la estadística o la econometría, a través de una conexión disciplinar que permite disponer de marcos históricos, conceptuales e instrumentales para desarrollar una investigación aplicada".

(4) Como señala Martínez González-Tablas (2008:396): "Sus oficiantes son zapadores de galerías ignotas, topos de mundos obscuros, buscadores de lo oculto, a veces, perdidos, como no puede menos de ser previsible, dada la dificultad desmesurada de su empeño".

(5) Probablemente el término keynesianismo sea uno de los más discutidos en la disciplina económica. La interpretación amplia que propone la Review of Keynesian Economics incluye: keynesianismo tradicional, el keynesianismo fundamentalista, el neo-keynesianismo, poskeynesianismo, keynesianismo sraffiano, keynesianismo kaleckiano, y el keynesianismo marxista. Propone que (a) la producción y el empleo están limitados por la demanda agregada, (b) considera que la problemática de la escasez de la demanda agregada existe independientemente de las rigideces nominales y (c) rechaza la afirmación de que el salario real es igual a la desutilidad marginal del trabajo.

\section{Bibliografía}

Akerman, J.G. 1962. Estructuras y ciclos económicos. Madrid: Ediciones Aguilar.

Alburquerque, F. 1981. El análisis dialéctico estructural de la realidad económica. Madrid: Galpa.

Álvarez, S. et.al. 2012. Por una economía inclusiva. Hacia un paradigma sistémico. Revista de Economía Crítica 14: 277-301. http://revistaeconomiacritica.org/sites/default/files/revistas/n14/Intervenciones-2.varios.pdf

Beiras, X. M. 1987. La teoría estructural de J. L. Sampedro. En: J. L. Sampedro et. al. Homenaje al profesor Sampedro: ciclo de conferencias. Madrid: Fundación Banco Exterior, pp. 57-78.

Berzosa, C. 1995. ¿Réquiem por el análisis de la estructura económica? En: Fernández, X. Estudios en homenaxe ao profesor Xosé Manuel Beiras Torrado. Santiago de Compostela: Universidade de Santiago de Compostela, pp. 33-49.

Gordon, D.M. 1995. Growth, distribution, and the rules of the game: social structuralist macro foundations for a democratic economic policy. En: G.A. Epstein y H. M. Gintis (eds.). Macroeconomic policy after the conservative era. Cambridge: Cambridge University Press, pp. 335-384.

Keynes, J. M. 1936. The general theory of employment, interest and money. London: Macmillan. 
Lawson, T. 1997. Economics and Reality. New York: Routledge.

Marglin, S.A. y Schor, J.B. 1990. The golden age of capitalism. Reinterpreting the postwar experience. New York: Clarendon Press.

Martínez González-Tablas, A. 2008. El análisis estructural y sus relaciones con el análisis sistémico y los análisis parciales. Revista de Economía Mundial 18: 393-404.

http://www.redalyc.org/articulo.oa?id=86601831

Palazuelos, E. 2000. Contenido y método de la economía. El análisis de la economía mundial. Madrid: Akal.

Palazuelos, E. 2015. Economía política mundial. Madrid: Akal.

Ramos, A. 2003. La explicación histórica como una modalidad de la economía aplicada. En: VV.AA. Estudios de historia de pensamiento económico: homenaje al profesor Francisco Bustelo García del Real. Madrid: Editorial Universidad Complutense, pp. 409-442.

Sampedro, J. L. 1959. Realidad económica y análisis estructural. Madrid: Aguilar.

Sampedro, J. L. 1983. El reloj, el gato y Madagascar. Revista de Estudios Andaluces 1: 119-126.

http://institucional.us.es/revistas/andaluces/1/art_9.pdf

Sampedro, J. L. 2010. Economía humanista: algo más que cifras. Madrid: Debate.

Sampedro, J. L. y Martínez Cortiña, R. 1973. Estructura económica. Teoría básica y estructura mundial. Barcelona: Ariel.

Viñas, A. 1976. Ciencia económica y análisis estructural. Revista Española de Economía 6(2): 101-146.

Recibido el 2 Mar 2015

Aceptado el 14 Jun 2015 